D.T. Wickramasinghe, L. Ferrario, and G.V. Bicknell, eds.

\title{
Photometric Evidence for the Presence of An Accretion Disk and for a Superhump - Like Phenomenon in the Classical Nova V1974 Cygni 1992
}

\author{
A. Retter, E.M. Leibowitz, and E.O. Ofek
}

School of Physics and Astronomy and the Wise Observatory, Raymond and Beverly Sackler Faculty of Exact Sciences, Tel-Aviv University, Israel

\begin{abstract}
The light curve of V1974 Cyg shows two distinct periodicities. The shorter periodicity is clearly the orbital period of the binary system. We show that the longer variation has similar features to permanent superhumps. This result indicates the existence of an accretion disk in the system no later than 30 months after the nova outburst. We used the precessing disk model of the superhump phenomenon and previous results in order to estimate that the white dwarf mass is at the range 0.75 $-1.07 \mathrm{M}_{\odot}$.
\end{abstract}

We present results of continuous photometry of V1974 Cygni performed during 32 nights in 1994 and 1995. The light curve (LC) of this classical nova shows a remarkable similarity to the superhump phenomenon, found in the SU Ursae Majoris subgroup of dwarf novae. This resemblance is expressed by the following facts:

1. The power spectrum of the LC shows clearly the presence of two distinct periodicities: $0.0812585 \mathrm{day} \approx 1.95$ hours and $0.0849767 \mathrm{~d} \approx 2.04 \mathrm{hr}$. (Semeniuk et al. 1995, Retter, Ofek \& Leibowitz 1995).

2. The two periods obey exactly the relation that holds between the orbital and superhump periods in the SU UMa members. (Stolz \& Schoembs 1981, 1984; Retter et al. 1995, Retter, Leibowitz \& Ofek 1996)

3. We establish the coherence of the shorter periodicity, and hence the likelihood that it is the orbital period of the binary system.

4. The peak to peak amplitude of the variation in the longer period is about 0.055 mag. The structure of its photometric cycle is similar to permanent superhumps (e.g. Patterson et al. 1993, Patterson and Skillman 1994).

5. The longer period oscillates around a certain value. The average $\dot{P}$ between times, in which $\mathrm{P}$ changes its sign is about $|\dot{P}| \sim 3 \times 10^{-7}$ - typical to permanent superhumps. (Patterson \& Skillman 1994.)

6 . We found that the harmonics of the main frequency are slightly displaced towards higher frequencies. This effect is reported in a few superhumpers (Patterson et al. 1995a and Harvey \& Patterson 1995).

7. A third periodicity of $0.083204 \mathrm{~d} \approx 2.00 \mathrm{hr}$ appeared in 1994 but not in 1995. It may be related to the recently discovered anti - superhump phenomenon (Patterson et al. 1993, Harvey et al. 1995, Patterson 1995 and Patterson et al. 1995a). 
The explanation of the longer period as caused by the rotation of the magnetic white dwarf (Semeniuk et al. 1995) is inconsistent with the fact that the system does not evolve towards synchronization. In particular, in summer 1995 it went against the trend expected in the nearly synchronous interpretation, it has lengthened rather than becoming shorter.

With one exception, RX J1940.1-1025, all polar systems have spin periods shorter than the orbital periods. (e.g. Patterson 1994). Even RX J1940.1-1025 has only a small period excess $(0.3 \%)$, while in V1974 Cyg the period excess is $4.6 \%$.

We therefore believe that our results, obtained over more than two years, indicate that the nova is in a permanent superhump stage. Our interpretation of the LC implies in particular, that V1974 Cyg contained an accretion disk less than 30 months after the nova outburst.

From the precessing disk model of the superhump phenomenon (Osaki, 1985) we estimate, that the mass ratio of the binary system is between 2.2 and 3.6. Combining with previous results (Paresce et al. 1995) this implies:

$0.75 \leq M_{W D} / M_{\odot} \leq 1.07$ and $0.19 \leq q \leq 0.28$.

\section{References}

Harvey D.A., Patterson J., 1995, PASP, 107, 1055.

Osaki Y., 1985, A\&A, 144, 369.

Paresce F., Livio M., Hack W., Korista K., 1995, A\&A, 299, 823.

Patterson J., 1994, PASP, 106, 209.

Patterson J., 1995, PASP, 107, 657.

Patterson J, Jablonski F., Koen C., O'Donghue D., Skillman D.R., 1995a, PASP, $107,1183$.

Patterson J., Skillman D.R., 1994, PASP, 106, 1141.

Patterson J., Skillman D.R., Thorstensen J.R., Hellier C., 1995b, PASP, 107, 307.

Patterson J. Thomas G.R., Skillman D., Diaz M., Suleimanov V.F., 1993, Ap.J.Suppl., $86,235$.

Retter A. Leibowitz E.M., Ofek E.O., 1996, to be published in the proceedings of the Keele conference, ed. Evans A. and Wood J.

Retter A., Ofek E.O., Leibowitz E.M., 1995, IAUC, 6158.

Semeniuk I., DeYoung J.A. Pych W., Olech A., Ruszkowski M., Schmidt R.E., 1995, Acta Astron., 45, 365.

Patterson J., Skillman D.R., 1994, PASP, 106, 1141.

Stolz B., Schoembs R., 1981, IBVS, 2029.

Stolz B., Schoembs R., 1984, A\&A, 132, 187. 